\title{
Environmental Fate - Geoökologie in einer sich verändernden Umwelt
}

\author{
Erste Ankündigung des GeoöKon 2009, Braunschweig, 04. bis 06. Dezember 2009
}

\author{
Wolfgang Durner $\cdot$ Andreas Horn
}

Erhalten: 7. Juni 2009/Akzeptiert: 8. Juni 2009/Online veröffentlicht: 18. Juni 2009

(C) Springer-Verlag 2009

Das Thema dieses GeoöKon hat vielfältige Aspekte. Environmental Fate im geoökologischen Kontext kann übersetzt und interpretiert werden als „Schicksal“, welches Substanzen in der Umwelt erfahren: Was passiert mit einer Substanz - wie wirkt die Umwelt auf die Substanz? Im engen Sinn verstehen wir darunter den Transport, die Verteilung auf die Kompartimente, Umwandlungen und Abbau. Wir können die Frage aber auch weiter fassen: Was bewirken Menschen und ihre Verhaltensweisen in der Umwelt? Kommt es zu Substanzanreicherungen oder Abreicherungen und sind die damit verbundenen Veränderungen aus Sicht des Menschen günstig oder ungünstig?

Beide Aspekte sind untrennbar miteinander verwoben, und in der Umweltsystemanalyse versuchen wir, den Weg von Substanzen in der Umwelt und die Folgen für die Umwelt $\mathrm{zu}$ erkennen, $\mathrm{zu}$ verfolgen (Monitoring) und $\mathrm{zu}$ beschreiben (Modeling). Die Geoökologie hat dabei als Zielsetzung, Probleme im Zusammenhang mit der menschlichen Nutzung zu erkennen und zu lösen und somit die Grundlage für eine nachhaltige Existenz der Menschen auf dem Globus zu schaffen.

Nach 20 Jahren geoökologischer Forschung und Ausbildung in Braunschweig kann Environmental Fate aber auch noch in einem weiteren, persönlichen Sinn interpretiert werden: Was wurde aus den jungen Menschen, die

W. Durner

Institut für Geoökologie,

Langer Kamp 19c, 38106 Braunschweig, Deutschland

E-Mail: w.durner@tu-bs.de

A. Horn $(\bowtie)$

Verband für Geoökologie in Deutschland (VGöD) e.V.,

Alexanderstr. 9, 95444 Bayreuth, Deutschland

E-Mail: andreas.horn@geooekologie.de hier (aus)gebildet wurden, die hier gearbeitet und gewirkt haben? Was hat die Umwelt an Lebensschicksal bereitgehalten, und wie beeinflusst ihr Wirken jetzt das Schicksal der Umwelt?

Es ist reizvoll und unsere Absicht, diese Facetten des Environmental Fate am GeoöKon 2009 im Rahmen von „20 Jahre Geoökologie in Braunschweig“ in einer JubiläumsVortragsreihe zu beleuchten.

\section{Vorläufiges Programm}

Die VGöD-Tagung beginnt am Freitag, den 4.12.2009 mit einem öffentlichen Abendvortrag von Prof. Dr. Hans-Rudolf Bork (Ökologie-Zentrum, Christian-Albrechts-Universität zu Kiel). Tags darauf (Samstag) sind zwei ganztägige Parallelveranstaltungen vorgesehen: ein Wissenschaftsforum und ein Praxisforum. Das Wissenschaftsforum wird von den Braunschweiger Professoren und weiteren eingeladenen Fachkolleginnen und -kollegen geleitet. Im Praxisforum berichten Geoökologinnen und Geoökologen über ihre Erfahrungen aus der Berufspraxis. Bei diesen Vorträgen kann es sich um Selbstpräsentationen, die Darstellung möglicher Arbeitsfelder oder auch um Themen mit Fortbildungscharakter handeln. Weiterhin findet am Samstag die Jahreshauptversammlung des VGöD statt sowie als feierlichgeselliger Ausklang ein Festakt anlässlich des 20-jährigen Bestehens der Geoökologie in Braunschweig. Am Sonntag wird der GeoöKon 2009 mit einem Exkursionsprogramm abgeschlossen.

Der VGöD empfiehlt allen Mitgliedern, sich die Tagung nicht entgehen zu lassen!

Weitere Informationen werden in Kürze auf der VGöDHomepage zu finden sein (www.geooekologie.de). 